\title{
Uniform Asymptotic Expansions of the Incomplete Gamma Functions and the Incomplete Beta Function
}

\author{
By N. M. Temme
}

\begin{abstract}
New asymptotic expansions are derived for the incomplete gamma functions and the incomplete beta function. In each case the expansion contains the complementary error function and an asymptotic series. The expansions are uniformly valid with respect to certain domains of the parameters.
\end{abstract}

1. Introduction. The incomplete gamma functions are defined by

$$
\gamma(a, x)=\int_{0}^{x} e^{-t} t^{a-1} d t, \quad \Gamma(a, x)=\int_{x}^{\infty} e^{-t} t^{a-1} d t .
$$

The parameters may be complex; but here we suppose $a$ and $x$ to be real, where $a>0$ and $x \geqslant 0$. Auxiliary functions are

$$
P(a, x)=\gamma(a, x) / \Gamma(a), \quad Q(a, x)=\Gamma(a, x) / \Gamma(a),
$$

and from the definitions it follows that

$$
\gamma(a, x)+\Gamma(a, x)=\Gamma(a), \quad P(a, x)+Q(a, x)=1 .
$$

For large values of $x$ we have the well-known asymptotic expansion,

$$
\Gamma(a, x) \sim x^{a-1} e^{-x}\left\{1+(a-1) / x+(a-1)(a-2) / x^{2}+\ldots\right\} .
$$

See for instance Dingle [1] or Olver [3]. If both $x$ and $a$ are large, this expansion is not useful, unless $a=o(x)$. For large values of $a$, we can better use the function $\gamma(a, x)$. From (1.1) we obtain the elementary result

$$
\gamma(a, x)=e^{-x} x^{a} \Gamma(a) \sum_{n=0}^{\infty} x^{n} / \Gamma(a+n+1) .
$$

This series converges for every finite $x$. It is useful for $a \rightarrow \infty$ and $x=o(a)$, since under this condition the series has an asymptotic character.

Expansions with a more uniform character are given by Tricomi [4], who found among others

$$
\begin{gathered}
\gamma\left(a+1, a+y(2 a)^{1 / 2}\right) / \Gamma(a+1)=1 / 2 \operatorname{erfc}(-y)-\frac{1}{3}(2 / a \pi)^{1 / 2}\left(1+y^{2}\right) \exp \left(-y^{2}\right) \\
+O\left(a^{-1}\right), \quad y, a \text { real, } a \rightarrow+\infty .
\end{gathered}
$$

This expansion is uniformly valid in $y$ on compact intervals of $\mathbf{R}$. The function erfc is the complementary error function defined by

Received May 9, 1974; revised December 1, 1974.

AMS (MOS) subject classifications (1970). Primary 33A15, 41 A60.

Key words and phrases. Incomplete gamma function, incomplete beta function, asymptotic expansion, error function. 


$$
\operatorname{erfc}(x)=2 \pi^{-1 / 2} \int_{x}^{\infty} e^{-t^{2}} d t
$$

It is a special case of $\Gamma(a, x)$, namely $\operatorname{erfc}(x)=\pi^{-1 / 2} \Gamma\left(1 / 2, x^{2}\right)$. Some results of Tricomi are corrected and used by Kölbig [2] for the construction of approximations of the zeros of the incomplete gamma function $\gamma(a, x)$.

An important book with many results on asymptotic expansions of the incomplete gamma functions is the recent treatise of Dingle [1]. Apart from elementary expansions, Dingle gives also uniform expansions and, in particular, he generalizes the results of Tricomi (p. 249 of [1]). Dingle does not specify the term "uniform", but it can be verified that the same restrictions on $y$ must hold as for (1.4).

In Section 2 we give new asymptotic expansions for $\gamma(a, x)$ and $\Gamma(a, x)$, holding uniformly in $0 \leqslant x / a$ for $a \rightarrow \infty$ and/or $x \rightarrow \infty$. In Section 3 an analogous result for the incomplete beta function is given.

A recent result of Wong [5] may be connected with our results. Wong considers integrals of which the endpoint is near by a saddle point of the integrand, and he applies his methods to the function $S_{n}(x)$ defined by

$$
e^{n x}=\sum_{r=0}^{n}(n x)^{r} / r !+(n x)^{n} S_{n}(x) / n !
$$

The function $S_{n}$ is a special case of the incomplete gamma function and the asymptotic expansion of $S_{n}(x)$ for $n \rightarrow \infty, x \sim 1$ is expressed by Wong in terms of the error function. (Wong interpreted his results only for $0 \leqslant x \leqslant 1$, but not across the transition point at $x=1$.)

2. Uniform Asymptotic Expansions. The integrals (1.1) are not attractive for deriving uniform expansions. Therefore, we write $P$ as

$$
P(a, x)=\frac{1}{2 \pi i} \int_{c-i \infty}^{c+i \infty} e^{x s} s^{-1}(s+1)^{-a} d s, \quad c>0
$$

in which $(s+1)^{-a}$ will have its principal value which is real for $s>-1$. Formula (2.1) can be found in Dingle's book. Here we derive it by observing that the Laplace transform of $d P(a, x) / d x$ is $(s+1)^{-a}$, from which it follows that $(s+1)^{-a} s^{-1}=$ $L(P(a, \cdot))$, which can be inverted to obtain (2.1). Taking into account the residue at $s=0$, the contour in (2.1) can be shifted to the left of the origin; and so a similar integral for $Q$ can be given. With (1.3) and some further modifications we arrive at

where

$$
Q(a, x)=\frac{e^{-a \phi(\lambda)}}{2 \pi i} \int_{c-i \infty}^{c+i \infty} e^{a \phi(t)} \frac{d t}{\lambda-t}, \quad 0<c<\lambda,
$$

$$
\phi(t)=t-1-\ln t, \quad \lambda=x / a .
$$

The contour in (2.2) will be deformed into a path in the s-plane which crosses the saddle point of the integrand. The saddle point $t_{0}$ follows from $\phi^{\prime}\left(t_{0}\right)=0$. Hence $t_{0}=1, \phi\left(t_{0}\right)=\phi^{\prime}\left(t_{0}\right)=0$ and $\phi^{\prime \prime}\left(t_{0}\right)=1$.

The steepest descent path follows from $\operatorname{Im} \phi(t)=\operatorname{Im} \phi\left(t_{0}\right)=0$, and, by writing $t=\sigma+i \tau(\sigma, \tau \in \mathbf{R})$ we obtain

$$
\sigma=\tau \operatorname{ctg} \tau, \quad-\pi<\tau<\pi .
$$


Let temporarily $\lambda>1$, that is $x>a$. Then the contour in (2.2) may be shifted into the contour $L$ in the $t$-plane defined by (2.4). According to Cauchy's theorem, the integral in (2.2) remains unaltered; and on $L$, the values of $\phi(t)$ are real and negative. Next we define the mapping of the $t$-plane into the $u$-plane by the equation,

$$
-1 / 2 u^{2}=\phi(t)
$$

with the condition $t \in L$ corresponds with $u \in \mathbf{R}$, and $u<0$ if $\tau<0, u>0$ if $\tau>0$.

The result is

$$
Q(a, x)=\frac{e^{-a \phi(\lambda)}}{2 \pi i} \int_{-\infty}^{\infty} e^{-1 / 2 a u^{2}} \frac{d t}{d u} \frac{d u}{\lambda-t}, \quad \lambda>1 .
$$

The presence of the pole at $t=\lambda$ in the integrand of (2.6) is somewhat disturbing, but we will get rid of it by writing

$$
\frac{d t}{d u} \frac{1}{\lambda-t}=\frac{d t}{d u} \frac{1}{\lambda-t}+\frac{1}{u-u_{1}}-\frac{1}{u-u_{1}}
$$

where $u_{1}$ is the point in the $u$-plane corresponding to the point $t=\lambda$ in the $t$-plane. That is, $-1 / 2 u_{1}^{2}=\phi(\lambda)$, hence $u_{1}= \pm i \phi(\lambda)^{1 / 2}$. There still is an ambiguity in the sign. However, the correct sign follows from the conditions imposed on the mapping defined in (2.5). In fact, we have

$$
u_{1}=i(1-\lambda)\left\{2(\lambda-1-\ln \lambda) /(1-\lambda)^{2}\right\}^{1 / 2},
$$

where the square root is positive for positive values of the argument. The first two terms at the right-hand side of (2.7) constitute a regular function at $t=\lambda$, and with this partition we obtain

$$
\begin{aligned}
& Q(a, x)=-\frac{e^{-a \phi(\lambda)}}{2 \pi i} \int_{-\infty}^{\infty} e^{-1 / 2 a u^{2}} \frac{d u}{u-u_{1}}+R(a, x), \\
& R(a, x)=\frac{e^{-a \phi(\lambda)}}{2 \pi i} \int_{-\infty}^{\infty} e^{-1 / 2 a u^{2}}\left\{\frac{d t}{d u} \frac{1}{\lambda-t}+\frac{1}{u-u_{1}}\right\} d u,
\end{aligned}
$$

and the integral in (2.9) can be expressed in terms of the complementary error function defined in (1.5), so that

$$
Q(a, x)=1 / 2 \operatorname{erfc}\left(a^{1 / 2} \zeta\right)+R(a, x), \quad \zeta=i u_{1} 2^{-1 / 2} .
$$

From $\operatorname{erfc}(x)+\operatorname{erfc}(-x)=2$ it follows that

$$
P(a, x)=1 / 2 \operatorname{erfc}\left(-a^{1 / 2} \zeta\right)-R(a, x) .
$$

So far, the results in (2.11) and (2.12) are exact, since no approximations were used. In order to obtain asymptotic expansions for $P(a, x)$ and $Q(a, x)$, the function $R(a, x)$ will be expanded in an asymptotic series. The integrand of $R(a, x)$ is a holomorphic function in the finite $u$-plane for every $\lambda \geqslant 0$. If we put the expansion,

$$
\frac{d t}{d u} \frac{1}{\lambda-t}+\frac{1}{u-u_{1}}=\sum_{k=0}^{\infty} c_{k}(\lambda) u^{k}
$$

in (2.10), and, if we reverse the order of summation and integration, by Watson's lemma [3], we obtain the expansion 


$$
R(a, x) \sim \frac{e^{-a \phi(\lambda)}}{2 \pi i} \sum_{k=0}^{\infty} c_{2 k}(\lambda) \Gamma(k+1 / 2)(1 / 2 a)^{-k-1 / 2} .
$$

(The conditions for Watson's lemma are certainly satisfied since the function in (2.13) is bounded for large real values of $u$.)

Each coefficient $c_{k}(\lambda)$ is an analytic function of $\lambda$ near $\lambda=1$, and the expansion (2.14) is not only valid near $\lambda=1$ but for all $\lambda \geqslant 0$. That is to say, we can fix $x$ and let $a$ tend to infinity, or conversely. Also, $x$ and $a$ may grow dependently or independently of each other.

The first few coefficients are

$$
\begin{aligned}
& c_{0}(\lambda)=\frac{i}{\lambda-1}-\frac{1}{u_{1}}, \quad c_{0}(1)=-\frac{i}{3}, \\
& c_{2}(\lambda)=\frac{-i\left(\lambda^{2}+10 \lambda+1\right)}{12(\lambda-1)^{3}}-\frac{1}{u_{1}^{3}}, \quad c_{2}(1)=-\frac{i}{540} .
\end{aligned}
$$

Our expansion is more powerful than those of Tricomi and Dingle. Tricomi's formula (1.4) follows from our expansion by expanding (2.12) for small values of $1-\lambda$. Moreover, for the complete expansion, Tricomi and Dingle obtained an infinite series, of which each term contains functions related to the error function. In our expansion, the information about the nonuniform behavior of the incomplete gamma functions is contained in just one error function. Besides, we obtain expansions for both $P$ and $Q$. Of course, the coefficients $c_{2 k}(\lambda)$ in (2.14) are more complicated than the coefficients in the other expansions.

As remarked before, the expansion (2.15) is also valid for fixed $a$ and $x \rightarrow \infty$, in spite of the nature of the series containing terms with negative powers of $a$. The coefficients, however, depend on $x$ and $a$; and, in fact, we can say that the sequence $\left\{d_{k}\right\}$, $d_{k}=c_{2 k}(\lambda) a^{-k}$, is an asymptotic sequence. That is, $d_{k+1}=o\left(d_{k}\right)$ if one (or both) of the parameters $a$ and $x$ is (are) large uniformly in $x / a \geqslant 0$.

3. The Incomplete Beta Function. The incomplete beta function is defined by

$$
I_{x}(p, q)=\frac{1}{B(p, q)} \int_{0}^{x} t^{p-1}(1-t)^{q-1} d t
$$

with $\operatorname{Re} p>0, \operatorname{Re} q>0,0 \leqslant x \leqslant 1$, and

$$
B(p, q)=\Gamma(p) \Gamma(q) / \Gamma(p+q) .
$$

The function in (3.2) is called the beta function. Again, we consider real variables $x$, $p$ and $q$, and we will derive an asymptotic expansion of $I_{x}(p, q)$ for large $p$ and $q$ uniformly valid for $0<\delta \leqslant x \leqslant 1$.

We first give an integral representation of $I_{x}$ which resembles those for the incomplete gamma function. Formula (3.1) is equivalent to

$$
I_{x}(p, q)=\frac{1}{B(p, q)} \int_{-\ln x}^{\infty} e^{-p t}\left(1-e^{-t}\right)^{q-1} d t
$$

and also, we have

$$
B(p, q)=\int_{0}^{\infty} e^{-p t}\left(1-e^{-t}\right)^{q-1} d t
$$


from which follows, by using the same technique as in the foregoing section,

$$
I_{x}(p, q)=\frac{1}{2 \pi i B(p, q)} \int_{c-i \infty}^{c+i \infty} e^{(p-s) \ln x} \frac{B(s, q)}{p-s} d s, \quad 0<c<p .
$$

This expression can be written as

$$
I_{x}(p, q)=\frac{x^{p}(1-x)^{q}}{2 \pi i} \frac{\Gamma(q) e^{q} q^{-q}}{B(p, q)} \int_{c-i \infty}^{c+i \infty} e^{q \psi(t)} F_{q}(t) \frac{d t}{t_{1}-t}
$$

with $\psi(t)=t \ln (t / x)-(1+t) \ln (1+t)-\ln (1-x)$,

$$
F_{q}(t)=\frac{\Gamma(q t) e^{q t}(q t)^{-q t}}{\Gamma(q+q t) e^{q(1+t)}(q+q t)^{-q(1+t)}}, \quad t_{1}=p / q, \text { and } 0<c<t_{1} .
$$

$F_{q}(t)$ is a slowly varying function as $q \rightarrow \infty$, on compact subsets of $|\arg t|<\pi, t \neq 0$. Of course, its construction is based on the Stirling approximation of the gamma function. For $\operatorname{larg} t \mid<\pi, t \neq 0$, we have

$$
F_{q}(t)=\{(1+t) / t\}^{1 / 2}\left(1+O\left(q^{-1}\right)\right), \quad q \rightarrow \infty, t \text { fixed }
$$

With

$$
t_{0}=x /(1+x), \quad x_{0}=p /(p+q),
$$

$t_{0}$ is a saddle point of $\psi$, and if $x=x_{0}$, this saddle point coincides with the pole at $t_{1}$.

The calculation of the saddle point $t_{0}$ is based on the assumption that the gamma functions in $F_{q}(t)$ in (3.6) have large arguments. Hence, for small values of $x$, which correspond to small values of $t_{0}$, the calculation is based on false assumptions. Therefore we only consider positive values of $x$. It is not necessary to have a uniform bound from zero of $x$. We are even allowing those values of $x$ with $q x \rightarrow \infty$.

From now on, details will be omitted, since the method is exactly the same as the one used in the foregoing section. We put $-1 / 2 u^{2}=\psi(t)$ and the results are

$$
\begin{gathered}
I_{x}(p, q)=1 / 2 \operatorname{erfc}\left(-(q / 2)^{1 / 2} \eta\right)+S_{x}(p, q), \\
\eta=\left(x-x_{0}\right)\left[2 q^{-1}\left\{p \ln \left(x_{0} / x\right)+q \ln \left(\left(1-x_{0}\right) /(1-x)\right)\right\} /\left(x-x_{0}\right)^{2}\right]^{1 / 2} .
\end{gathered}
$$

The square root is positive for positive values of its argument. The function $S_{x}$ is defined by

$$
\begin{gathered}
S_{x}(p, q)=\frac{x^{p}(1-x)^{q} \Gamma(q) e^{-q} q^{q}}{2 \pi i B(p, q)} \int_{-\infty}^{\infty} e^{-1 / 2 q u^{2}} G(u) d u, \\
G(u)=\frac{F_{q}(t)}{t_{1}-t} \frac{d t}{d u}+\frac{F_{q}\left(t_{1}\right)}{u-u_{1}} .
\end{gathered}
$$

For $u_{1}$ we have $-1 / 2 u_{1}^{2}=\psi\left(t_{1}\right), u_{1}=i \eta$.

The role of the parameter $\lambda$ of the foregoing section is now played by $\left(x-x_{0}\right)$. We have

$$
\eta=-\frac{p+q}{q}\left(\frac{p+q}{p}\right)^{1 / 2}\left(x-x_{0}\right)\left\{1+\frac{1}{3} \frac{p^{2}-q^{2}}{p q}\left(x-x_{0}\right)+O\left(x-x_{0}\right)^{2}\right\}
$$


for $x \rightarrow x_{0}$. An asymptotic expansion of $S_{x}$ is obtained by expanding $G(u)=\Sigma d_{k} u^{k}$, giving

$$
\begin{gathered}
S_{x}(p, q) \sim \frac{x^{p}(1-x)^{q}}{2 \pi i} \frac{\Gamma(p+q)}{\Gamma(p)} e^{q} q^{-q} \sum_{k=0}^{\infty} d_{2 k} \Gamma(k+1 / 2)(1 / 2 q)^{-k-1 / 2}, \\
d_{0}=i \frac{F_{q}\left(t_{0}\right)}{t_{1}-t_{0}} \frac{x^{1 / 2}}{1-x}-\frac{F_{q}\left(t_{1}\right)}{u_{1}} .
\end{gathered}
$$

The expansion holds for $p \rightarrow \infty$ and/or $q \rightarrow \infty$, uniformly in $\delta \leqslant x \leqslant 1$, where $\delta$ may depend on $q$, such that $q \delta \rightarrow \infty$.

A more transparent first approximation for $S_{x}(p, q)$ is obtained by replacing the functions $F_{q}$ in (3.14) by the approximation (3.7). The result is

$$
\begin{aligned}
S_{x}(p, q)= & \{p /[2 \pi q(p+q)]\}^{1 / 2}\left(x / x_{0}\right)^{p}\left\{(1-x) /\left(1-x_{0}\right)\right\}^{q} \\
& \cdot\left\{\left(1-x_{0}\right) /\left(x_{0}-x\right)+\eta^{-1} x_{0}^{-1 / 2}\right\}\left(1+O\left(q^{-1}\right)\right), \quad q \rightarrow \infty .
\end{aligned}
$$

Department of Applied Mathematics

Mathematical Centre

Amsterdam, The Netherlands

1. R. B. DINGLE, Asymptotic Expansions: Their Derivation and Interpretation, Academic Press, London and New York, 1973.

2. K. S. KÖLBIG, "On the zeros of the incomplete gamma function," Math. Comp., v. 26, 1972, pp. $751-755$. MR 48 \#5336. York, 1974.

3. F. W. J. OLVER, Asymptotics and Special Functions, Academic Press, London and New

4. F. G. TRICOMI, "Asymptotische Eigenschaften der unvollständigen Gammafunktion," Math. Z., v. 53, 1950, pp. 136-148. MR 13, 553.

5. R. WONG, "On uniform asymptotic expansion of definite integrals," J. Approximation Theory, v. 7, 1973, pp. 76-86. 